\title{
On the number of variables in undecidable superintuitionistic propositional calculi
}

\author{
Grigoriy V. Bokov \\ Department of Mathematical Theory of Intelligent Systems \\ Lomonosov Moscow State University \\ Moscow, Russian Federation \\ E-mail: bokov@intsys.msu.ru \\ September 7, 2018
}

\begin{abstract}
In this paper, we construct an undecidable 3-variable superintuitionistic propositional calculus, i.e., a finitely axiomatizable extension of the intuitionistic propositional calculus with axioms containing only 3 variables. Since there are no 2 -variable superintuitionistic propositional calculi, this is the minimal possible number of variables.
\end{abstract}

\section{Introduction}

Decidability is the important property of propositional calculi, it means that the set of their derivable formulas (or theorems) can be effectively determined. A natural question is how to separate classes of decidable and undecidable calculi. On the other hand, since undecidable propositional calculi can be used as a base for obtaining "negative" results to various algorithmic problems, it is of interest to find the simplest possible calculus of that class. There are many possible ways to separate decidable and undecidable calculi. A significant and simplest way is to describe the number of variables in their axioms.

In 1949, Linial and Post [10] found the first undecidable propositional calculus. In 1975, Hughes and Singletary [9] proved that there is an undecidable propositional calculus with axioms containing 3 variables. In 1976, Hughes [8] constructed an undecidable implicational propositional calculus using axioms in 2 variables. Finally, Gladstone in 1979 [7] proved that every 1-variable propositional calculus is decidable.

The first undecidable superintuitionistic propositional calculus was built in 1978 by Shehtman [15, 16]. Axioms of this calculus contain 7 variables. Later Chagrov in 1994 [4] did the same using axioms with only 4 variables. In [5, Sections 16.9] he noted that it is unknown whether there exist undecidable superintuitionistic propositional calculi with axioms in 2 or 3 variables.

In [6] Gladstone proved that the following formula

$$
A=(p \rightarrow q) \rightarrow((q \rightarrow r) \rightarrow(p \rightarrow r))
$$


is not derivable from the set of all 2-variable tautologies by modus ponens and substitution. Since $A$ is an intuitionistic tautology, therefore a 2-variable propositional calculus cannot derive all intuitionistic tautologies. If we combine this with Gladstone's result for 1-variable propositional calculi, we get that there are no undecidable superintuitionistic propositional calculi with axioms containing less than 3 variables. The aim of this paper is to construct an undecidable 3-variable superintuitionistic propositional calculus.

This paper is organized as follows. In the next section we introduce the basic terminology and notation. In Section 3 we state and prove our main result. Finally, in Section 4 we give some concluding remarks and discuss further directions of research.

\section{Definitions}

In this section, we recall definitions of the intuitionistic propositional calculus and Kripke semantics. For more details we refer the reader to [5].

First, we introduce some notation. Let us consider the language consisting of an infinite set of propositional variables $\mathcal{V}$, brackets, and the signature $\Sigma=\{\perp, \wedge, \vee, \rightarrow\}$, where $\perp$ is the constant symbol, $\wedge, \vee$ and $\rightarrow$ are binary connectives. Letters $p, q, x, y$, etc., are used to denote propositional variables. We define $\neg, \leftrightarrow$ and $\top$ as the usual abbreviations: $\neg A:=A \rightarrow \perp, A \leftrightarrow B=(A \rightarrow B) \wedge(B \rightarrow A)$, and $\top=\neg \perp$.

Propositional formulas or $\Sigma$-formulas are built up from the signature $\Sigma$, propositional variables from $\mathcal{V}$, and brackets in the usual way. For example, the following notations

$$
x, \quad \neg A, \quad(A \wedge B), \quad(A \vee B), \quad(A \rightarrow B)
$$

are formulas if $A, B$ are formulas. Capital letters $A, B, C$, etc., are used to denote propositional formulas. Throughout the paper, we omit some parentheses in formulas whenever it does not lead to confusion.

By a propositional calculus or a $\Sigma$-calculus we mean a finite set $P$ of $\Sigma$-formulas referred to as axioms together with two rules of inference:

1) modus ponens

$$
A, A \rightarrow B \vdash B
$$

2) substitution

$$
A \vdash \sigma A,
$$

where $\sigma A$ is a substitution instance of $A$, i.e., the result of applying a substitution $\sigma$ to the formula $A$.

Denote by $[P]$ the set of derivable (or provable) formulas of a calculus $P$. A derivation in $P$ is defined from the axioms and the rules of inference in the usual way. The statement that a formula $A$ is derivable from $P$ is denoted by $P \vdash A$.

Let us introduce the following pre-order relation on the set of all propositional calculi. We write $P_{1} \leq P_{2}$ (or, equivalently, $P_{2} \geq P_{1}$ ) if each derivable formula of $P_{1}$ is also derivable from $P_{2}$, i.e., if $\left[P_{1}\right] \subseteq\left[P_{2}\right]$. We write $P_{1} \sim P_{2}$ and say that two calculi $P_{1}$ and $P_{2}$ are equivalent if $\left[P_{1}\right]=\left[P_{2}\right]$. Finally, we write $P_{1}<P_{2}$ if $\left[P_{1}\right] \subsetneq\left[P_{2}\right]$.

An intuitionistic Kripke frame is a pair $\mathfrak{F}=\langle W, R\rangle$ consisting of a nonempty set $W$ and a partial order $R$ on $W$, which is reflexive, transitive and antisymmetric, i.e., $\mathfrak{F}$ is just a partially ordered set. The elements of $W$ are called the points (or worlds) of the frame $\mathfrak{F}$, 
and the relation $R$ is called the accessibility relation. If for some $w, w^{\prime} \in W$ the relation $w R w^{\prime}$ holds, we say that $w^{\prime}$ is accessible from $w$ or $w$ sees $w^{\prime}$. We write $w \leq_{R} w^{\prime}\left(\right.$ or $\left.w^{\prime} \geq_{R} w\right)$ iff $w R w^{\prime}$.

A valuation in an intuitionistic frame $\mathfrak{F}=\langle W, R\rangle$ is a map $\mathfrak{V}$ associating with each propositional variable $p \in \mathcal{V}$ some (possibly empty) subset $\mathfrak{V}(p)$ of $W$ such that, for every $w \in \mathfrak{V}(p)$ and every $w^{\prime} \in W, w \leq_{R} w^{\prime}$ implies $w^{\prime} \in \mathfrak{V}(p)$.

An intuitionistic Kripke model is a pair $\mathfrak{M}=\langle\mathfrak{F}, \mathfrak{V}\rangle$, where $\mathfrak{F}$ is an intuitionistic frame and $\mathfrak{V}$ is a valuation in $\mathfrak{F}$.

Let $\mathfrak{M}=\langle\mathfrak{F}, \mathfrak{V}\rangle$ be an intuitionistic Kripke model and $w$ be a point in the frame $\mathfrak{F}=$ $\langle W, R\rangle$. By induction on the construction of a formula $A$ we define a relation $(\mathfrak{M}, w) \models A$, which is read as $A$ is true at $w$ in $\mathfrak{M}$ :

$$
\begin{array}{lll}
(\mathfrak{M}, w) \models \perp & \\
(\mathfrak{M}, w) \models p & \Longleftrightarrow & w \in \mathfrak{V}(p) ; \\
(\mathfrak{M}, w) \models A \wedge B & \Longleftrightarrow & (\mathfrak{M}, w) \models A \text { and }(\mathfrak{M}, w) \models B ; \\
(\mathfrak{M}, w) \models A \vee B & \Longleftrightarrow & (\mathfrak{M}, w) \models A \text { or }(\mathfrak{M}, w) \models B ; \\
(\mathfrak{M}, w) \models A \rightarrow B & \Longleftrightarrow & \text { for all } w^{\prime} \in W \text { such that } w \leq_{R} w^{\prime}, \\
& \left(\mathfrak{M}, w^{\prime}\right) \models A \text { implies }\left(\mathfrak{M}, w^{\prime} \models B .\right.
\end{array}
$$

From the definition it follows that

$$
\begin{aligned}
& (\mathfrak{M}, w) \models \top \\
& (\mathfrak{M}, w) \models \neg A \quad \Longleftrightarrow \quad \text { for all } w^{\prime} \in W \text { such that } w \leq_{R} w^{\prime},\left(\mathfrak{M}, w^{\prime}\right) \not \models A .
\end{aligned}
$$

If $(\mathfrak{M}, w) \models A$ does not hold, i.e., $(\mathfrak{M}, w) \not \models A$, we say that $A$ is refuted at the point $w$ in $\mathfrak{M}$.

We say that $A$ is valid in a model $\mathfrak{M}=\langle\mathfrak{F}, \mathfrak{V}\rangle$ defined on a frame $\mathfrak{F}=\langle W, R\rangle$ if $(\mathfrak{M}, w) \models A$ for all $w \in W$; if $A$ is valid in $\mathfrak{M}$, we write $\mathfrak{M} \models A$. We say that $A$ is valid in a frame $\mathfrak{F}=\langle W, R\rangle$ if $A$ is valid in every model based on $\mathfrak{F}$; if $A$ is valid in $\mathfrak{F}$, we write $\mathfrak{F} \models A$. We say that $A$ is true at a point $w$ in a frame $\mathfrak{F}$ if $(\mathfrak{M}, w) \models A$ for every model $\mathfrak{M}$ defined on $\mathfrak{F}$; if $A$ is true at the point $w$ in frame $\mathfrak{F}$, we write $(\mathfrak{F}, w) \models A$. If $\mathfrak{M}$ is fixed we write $w \models A$ instead of $(\mathfrak{M}, w) \models A$.

We define the intuitionistic propositional calculus Int as the smallest propositional calculus containing the following set of axioms:

$$
\begin{array}{ll}
\left(\neg_{1}\right) & p \rightarrow(q \rightarrow p) \\
\left(\neg_{2}\right) & (p \rightarrow(q \rightarrow r)) \rightarrow((p \rightarrow q) \rightarrow(q \rightarrow r)) \\
\left(\wedge_{1}\right) & p \wedge q \rightarrow p \\
\left(\wedge_{2}\right) & p \wedge q \rightarrow q \\
\left(\wedge_{3}\right) & p \rightarrow(q \rightarrow p \wedge q) \\
\left(\vee_{1}\right) & p \rightarrow p \vee q \\
\left(\vee_{2}\right) & q \rightarrow p \vee q \\
\left(\vee_{3}\right) & (p \rightarrow r) \rightarrow((q \rightarrow r) \rightarrow(p \vee q \rightarrow r)) \\
(\neg 1) & (p \rightarrow q) \rightarrow((p \rightarrow \neg q) \rightarrow \neg p) \\
(\neg 2) & p \rightarrow(\neg p \rightarrow q)
\end{array}
$$

It is well known that

$$
\text { Int } \vdash A \Longleftrightarrow \mathfrak{F} \models A \text {, for every Kripke frame } \mathfrak{F} \text {. }
$$


By a superintuitionistic propositional calculus we mean a propositional calculus obtained from Int by adding a finite set of new axioms. If $M$ is a finite set of propositional formulas, then a propositional calculus obtained from Int by adding new axioms $M$ is denoted by Int $+M$. Since

$$
\text { Int }+\left\{A_{1}, \ldots, A_{n}\right\} \sim \text { Int }+A_{1} \wedge \ldots \wedge A_{n}
$$

we can assume that a superintuitionistic propositional calculus is a calculus Int $+A$ for some intuitionistic propositional formula $A$.

\section{Main result}

Our main result is the following theorem.

Theorem 3.1. There is a 3-variable intuitionistic propositional formula $A$ such that Int $+A$ is undecidable.

First, we recall what a Minsky machine is and encode configurations of a Minsky machine by superintuitionistic propositional formulas. Next, we construct a Kripke model refuting all codes of derivable configurations. Finally, we encode instructions of a Minsky machine $\mathcal{M}$ by a single superintuitionistic formula $A_{\mathcal{M}}$ and formally reduce the configuration problem of $\mathcal{M}$ to the derivation problem of a superintuitionistic propositional calculus $\mathbf{I n t}+A_{\mathcal{M}}$.

\subsection{Minsky machine}

There are many algorithmic formalisms to prove the undecidability of a propositional calculus [3]. For example, the undecidability of a calculus contained in the classical [1], intuitionistic [2] propositional calculus or in another subcalculus [3] can be easily proved by using tag systems. But for extensions of the intuitionistic propositional calculus, this is very hard [12, 17]. For this reason, in order to prove the undecidability of superintuitionistic propositional calculi we will use an algorithmic formalism which is called Minsky machines [11]. In [5] Chagrov mentioned that it is the most convenient formalism for being simulated by modal and intuitionistic formulas.

In accordance with [5] we define a Minsky machine as a finite set of instructions for transforming triples $\langle s, m, n\rangle$ of natural numbers, called configurations, where $s$ is the number of the instruction to be executed at the next step (referred to as the current machine state), and $m, n \in \mathbb{N} 1$. Each instruction has one of the following four forms:

$$
\begin{aligned}
& s \mapsto\langle t, 1,0\rangle, \quad s \mapsto\langle t,-1,0\rangle /\langle u, 0,0\rangle, \\
& s \mapsto\langle t, 0,1\rangle, \quad s \mapsto\langle t, 0,-1\rangle /\langle u, 0,0\rangle,
\end{aligned}
$$

where $s, t, u$ are the machine states. Note that all Minsky machines are assumed to be deterministic, i.e., they may not contain distinct instructions with the same numbers.

As an example, let us consider the applying of first two instructions. The instruction

$$
s \mapsto\langle t, 1,0\rangle
$$

\footnotetext{
${ }^{1}$ We assume that $\mathbb{N}=\{0,1,2, \ldots\}$.
} 
transforms $\langle s, m, n\rangle$ into $\langle t, m+1, n\rangle$, and the instruction

$$
s \mapsto\langle t,-1,0\rangle /\langle u, 0,0\rangle
$$

transforms $\langle s, m, n\rangle$ into $\langle t, m-1, n\rangle$ if $m>0$ and into $(u, m, n)$ if $m=0$. The meaning of the others is defined analogously.

Let $\mathcal{M}$ be a Minsky machine, then the notation $\langle s, m, n\rangle \stackrel{\mathcal{M}}{\longmapsto}\langle t, k, l\rangle$ means that the configuration $\langle t, k, l\rangle$ is obtained from $\langle s, m, n\rangle$ by applying an instruction of machine $\mathcal{M}$ once. We write $\langle s, m, n\rangle \stackrel{\mathcal{M}}{\Longleftrightarrow}\langle t, k, l\rangle$ if the configuration $\langle t, k, l\rangle$ is obtained from $\langle s, m, n\rangle$ by applying instructions of machine $\mathcal{M}$ in finitely many steps (possibly, in 0 steps). Particularly, we always have $\langle s, m, n\rangle \stackrel{\mathcal{M}}{\Longleftrightarrow}\langle s, m, n\rangle$.

The configuration problem for a Minsky machine $M$ and a configuration $\langle s, m, n\rangle$ is, given a configuration $\langle t, k, l\rangle$, to determine whether $\langle s, m, n\rangle \stackrel{\mathcal{M}}{\Longleftrightarrow}\langle t, k, l\rangle$.

Theorem 3.2 (Minsky, [1]). There exist a Minsky machine $\mathcal{M}$ and a configuration $\langle s, m, n\rangle$ for which the configuration problem is undecidable.

Let $\mathcal{M}$ be a Minsky machine and $\left\langle s_{0}, m_{0}, n_{0}\right\rangle$ a configuration for which the configuration problem is undecidable.

\subsection{Encoding of configurations}

Let $p, q$ and $r$ be three distinct propositional variables. Now we define some propositional formulas using only variables $p, q, r$, which encode configurations of Minsky machines. Note that some basic ideas of defining these formulas was found in [5] and [13].

First, let us define the following groups of propositional formulas constructed from variables $p, q$ and $r$. If

$$
\begin{gathered}
S_{-2}[x]=\neg x, \quad S_{-1}[x]=T_{-2}[x] \rightarrow x, \\
T_{-2}[x]=\neg \neg x, \quad T_{-1}[x]=S_{-1}[x] \rightarrow S_{-2}[x] \vee T_{-2}[x], \\
S_{i}[x]=T_{i-1}[x] \rightarrow S_{i-1}[x] \vee T_{i-2}[x], \\
T_{i}[x]=\quad S_{i}[x] \rightarrow S_{i-1}[x] \vee T_{i-1}[x],
\end{gathered}
$$

for all $i \geq 0$, then we define

Groups $\left(A^{0}\right)$ and $\left(B^{0}\right)$ :

$$
A_{i}^{0}=S_{i+3}[r], \quad B_{i}^{0}=T_{i+3}[r] \quad \text { for all } i \geq-5 .
$$

Let $C_{1}=A_{0}^{0}$ and $C_{2}=B_{0}^{0}$, then

Groups $\left(A^{1}\right)$ and $\left(B^{1}\right)$ :

$$
\begin{gathered}
A_{i}^{1}=S_{i+3}[p], \quad B_{i}^{1}=T_{i+3}[p] \quad \text { for } i \in\{-3,-4,-5\}, \\
A_{-2}^{1}=B_{-3}^{1} \rightarrow A_{-3}^{1} \vee B_{-4}^{1}, \quad A_{-1}^{1}=B_{-2}^{1} \rightarrow A_{-2}^{1} \vee B_{-3}^{1}, \\
B_{-2}^{1}=A_{-3}^{1} \rightarrow C_{1} \vee B_{-3}^{1}, \quad B_{-1}^{1}=A_{-2}^{1} \rightarrow A_{-3}^{1} \vee B_{-2}^{1}, \\
A_{i}^{1}=C_{2} \wedge B_{i-1}^{1} \rightarrow C_{1} \vee A_{i-1}^{1} \vee B_{i-2}^{1}, \\
B_{i}^{1}=C_{2} \wedge A_{i-1}^{1} \rightarrow C_{1} \vee A_{i-2}^{1} \vee B_{i-1}^{1}, \quad \text { for all } i \geq 0 ;
\end{gathered}
$$


Groups $\left(A^{2}\right)$ and $\left(B^{2}\right)$ :

$$
\begin{gathered}
A_{i}^{2}=S_{i+3}[q], \quad B_{i}^{2}=T_{i+3}[q] \quad \text { for } i \in\{-3,-4,-5\}, \\
A_{-2}^{2}=B_{-3}^{2} \rightarrow A_{-3}^{2} \vee B_{-4}^{2}, \quad A_{-1}^{2}=B_{-2}^{2} \rightarrow A_{-2}^{2} \vee B_{-3}^{2}, \\
B_{-2}^{2}=A_{-3}^{2} \rightarrow C_{2} \vee B_{-3}^{2}, \quad B_{-1}^{2}=A_{-2}^{2} \rightarrow A_{-3}^{2} \vee B_{-2}^{2}, \\
A_{i}^{2}=C_{1} \wedge B_{i-1}^{2} \rightarrow C_{2} \vee A_{i-1}^{2} \vee B_{i-2}^{2}, \\
B_{i}^{2}=C_{1} \wedge A_{i-1}^{2} \rightarrow C_{2} \vee A_{i-2}^{2} \vee B_{i-1}^{2}, \quad \text { for all } i \geq 0 .
\end{gathered}
$$

Note that the groups $\left(A^{0}\right),\left(B^{0}\right)$ contain only variable $r,\left(A^{1}\right),\left(B^{1}\right)$ contain only variables $r$, $p$, and $\left(A^{2}\right),\left(B^{2}\right)$ contain only variables $r, q$. Now we define formulas encoding configurations of the Minsky machine $\mathcal{M}$.

Group $(E)$ :

$$
\begin{aligned}
E_{s, m, n}=A_{3 s+2}^{0} \wedge B_{3 s+2}^{0} \wedge A_{m+1}^{1} \wedge B_{m+1}^{1} \wedge A_{n+1}^{2} & \wedge B_{n+1}^{2} \rightarrow \\
& \rightarrow A_{3 s+1}^{0} \vee B_{3 s+1}^{0} \vee A_{m}^{1} \vee B_{m}^{1} \vee A_{n}^{2} \vee B_{n}^{2},
\end{aligned}
$$

for all $s, m, n \geq 0$. The formula $E_{s, m, n}$ is called the code of a configuration $\langle s, m, n\rangle$.

Denote by $(A)$ and $(B)$ the following sets of formulas:

$$
\begin{aligned}
& (A)=\left(A^{0}\right) \cup\left(A^{1}\right) \cup\left(A^{2}\right), \\
& (B)=\left(B^{0}\right) \cup\left(B^{1}\right) \cup\left(B^{2}\right),
\end{aligned}
$$

and by $M$ the set of formulas:

$$
M=(A) \cup(B) \cup(E) .
$$

\subsection{Kripke model refuting codes of derivable configurations}

In this section, we construct a Kripke model $\mathfrak{M}=\langle\mathfrak{F}, \mathfrak{V}\rangle$ refuting all formulas from $M$, i.e., for every formula from $M$, there exists a unique maximal point, at which this formula is refuted.

First, let us define the following equivalence relation $\sim_{\mathcal{M}}$ on the set of all configurations $\{\langle s, m, n\rangle \mid s, m, n \geq 0\}$ :

$$
\langle s, m, n\rangle \sim_{\mathcal{M}}\langle t, k, l\rangle \leftrightharpoons\langle s, m, n\rangle \stackrel{\mathcal{M}}{\Longrightarrow}\langle t, k, l\rangle \text { and }\langle t, k, l\rangle \stackrel{\mathcal{M}}{\Longleftrightarrow}\langle s, m, n\rangle .
$$

Denote by $[s, m, n]$ the equivalence class of a configuration $\langle s, m, n\rangle$ :

$$
[s, m, n]=\left\{\langle t, k, l\rangle \mid\langle s, m, n\rangle \sim_{\mathcal{M}}\langle t, k, l\rangle\right\} .
$$

The set of all equivalence classes of relation $\sim_{\mathcal{M}}$ is denoted by $\mathcal{E}_{\mathcal{M}}$.

Let us define the relation $\stackrel{\mathcal{M}}{\Longleftrightarrow}$ on the set of equivalence classes $\mathcal{E}_{\mathcal{M}}$ :

$$
[s, m, n] \stackrel{\mathcal{M}}{\Longleftrightarrow}[t, k, l] \leftrightharpoons\langle s, m, n\rangle \stackrel{\mathcal{M}}{\Longleftrightarrow}\langle t, k, l\rangle \text {. }
$$

Greek letters $\alpha, \beta, \gamma$, etc., are used to denote equivalence classes. Denote by $\alpha_{0}$ the equivalence class of the initial configuration $\left\langle s_{0}, m_{0}, n_{0}\right\rangle$, i.e., $\alpha_{0}=\left[s_{0}, m_{0}, n_{0}\right]$. 
Now we define a Kripke frame $\mathfrak{F}=\langle W, R\rangle$ as follows. Let

$$
\bigcup_{\substack{i \geq-5, j \in\{0,1,2\}}}\left\{a_{i}^{j}, b_{i}^{j}\right\} \cup \underset{\substack{\alpha \in \mathcal{E}_{\mathcal{M}}: \\ \alpha_{0} \stackrel{\mathcal{M}}{\Longrightarrow} \alpha}}{\bigcup}\left\{e_{\alpha}\right\}
$$

To define the accessibility relation $R$ on $W$, we consider the following groups of relations:

Group $R_{i}^{j}, i \geq-4, j \in\{0,1,2\}$ :

$$
\begin{aligned}
R_{-4}^{j} & =\left\{\left\langle a_{-4}^{j}, a_{-5}^{j}\right\rangle,\left\langle b_{-4}^{j}, a_{-5}^{j}\right\rangle,\left\langle b_{-4}^{j}, b_{-5}^{j}\right\rangle\right\}, \\
R_{-3}^{j} & =\left\{\left\langle a_{-3}^{j}, a_{-4}^{j}\right\rangle,\left\langle a_{-3}^{j}, b_{-5}^{j}\right\rangle,\left\langle b_{-3}^{j}, a_{-4}^{j}\right\rangle,\left\langle b_{-3}^{j}, b_{-4}^{j}\right\rangle\right\}, \\
R_{i}^{0} & =\left\{\left\langle a_{i}^{0}, a_{i-1}^{0}\right\rangle,\left\langle a_{i}^{0}, b_{i-2}^{0}\right\rangle,\left\langle b_{i}^{0}, a_{i-1}^{0}\right\rangle,\left\langle b_{i}^{0}, b_{i-1}^{0}\right\rangle\right\} \text { for all } i \geq-2 \text { and } \\
R_{-2}^{1} & =\left\{\left\langle a_{-2}^{1}, a_{-3}^{1}\right\rangle,\left\langle a_{-2}^{1}, b_{-4}^{1}\right\rangle,\left\langle b_{-2}^{1}, a_{0}^{0}\right\rangle,\left\langle b_{-2}^{1}, b_{-3}^{1}\right\rangle\right\}, \\
R_{-2}^{2} & =\left\{\left\langle a_{-2}^{2}, a_{-3}^{2}\right\rangle,\left\langle a_{-2}^{2}, b_{-4}^{2}\right\rangle,\left\langle b_{-2}^{2}, b_{0}^{0}\right\rangle,\left\langle b_{-2}^{2}, b_{-3}^{2}\right\rangle\right\}, \\
R_{i}^{j} & =\left\{\left\langle a_{i}^{j}, a_{i-1}^{j}\right\rangle,\left\langle a_{i}^{j}, b_{i-2}^{j}\right\rangle,\left\langle b_{i}^{j}, a_{i-2}^{j}\right\rangle,\left\langle b_{i}^{j}, b_{i-1}^{j}\right\rangle\right\} \text { for all } i \geq-1, j \in\{1,2\} ;
\end{aligned}
$$

Group $R_{s, m, n}, s, m, n \geq 0, \alpha_{0} \stackrel{\mathcal{M}}{\Longrightarrow}[s, m, n]$ :

$$
\begin{aligned}
R_{s, m, n}=\{ & \left\langle e_{[s, m, n]}, a_{3 s+1}^{0}\right\rangle,\left\langle e_{[s, m, n]}, b_{3 s+1}^{0}\right\rangle,\left\langle e_{[s, m, n]}, a_{m}^{1}\right\rangle, \\
& \left.\left\langle e_{[s, m, n]}, b_{m}^{1}\right\rangle,\left\langle e_{[s, m, n]}, a_{n}^{2}\right\rangle,\left\langle e_{[s, m, n]}, b_{n}^{2}\right\rangle\right\} .
\end{aligned}
$$

Let

$$
R^{\prime}=\bigcup_{\substack{i \geq-4, j \in\{0,1,2\}}} R_{i}^{j} \cup \bigcup_{\substack{s, m, n \geq 0: \\ \alpha_{0} \stackrel{\mathcal{M}}{\Longrightarrow}[s, m, n]}} R_{s, m, n} \cup \bigcup_{\substack{\alpha, \beta \in \mathcal{E}_{\mathcal{M}}: \\ \alpha \stackrel{\mathcal{M}}{\Longrightarrow} \beta}}\left\{\left\langle e_{\alpha}, e_{\beta}\right\rangle\right\} .
$$

We take as $R$ the reflexive and transitive closure of $R^{\prime}$.

Let us define a valuation $\mathfrak{V}$ of the Kripke model $\mathfrak{M}=\langle\mathfrak{F}, \mathfrak{V}\rangle$ in the following way:

$$
\begin{aligned}
& (\mathfrak{M}, w) \forall r \quad \Longleftrightarrow w \leq_{R} a_{-4}^{0} \text { or } w \leq_{R} b_{-5}^{0} ; \\
& (\mathfrak{M}, w) \forall=p \quad \Longleftrightarrow w \leq_{R} a_{-4}^{1} \text { or } w \leq_{R} b_{-5}^{1} ; \\
& (\mathfrak{M}, w) \forall \forall \quad \Longleftrightarrow w \leq_{R} a_{-4}^{2} \text { or } w \leq_{R} b_{-5}^{2} .
\end{aligned}
$$

The model $\mathfrak{M}$ is depicted on Figure 1. Now we prove some basic semantic properties of the Kripke model $\mathfrak{M}$.

Lemma 3.3. Let $w$ be a world of $\mathfrak{M}$, then

$$
\begin{aligned}
w \forall A_{i}^{j} & \Longleftrightarrow w \leq_{R} a_{i}^{j}, \\
w \forall B_{i}^{j} & \Longleftrightarrow w \leq_{R} b_{i}^{j}
\end{aligned}
$$

for all $i \geq-4$ and $j \in\{0,1,2\}$.

Proof. By induction on $i \geq-4$.

Induction base consists of the following cases:

1) $i=-4$. Let $x_{0}=r, x_{1}=p$, and $x_{2}=q$.

Since $w \forall x_{j}$ iff $w \leq_{R} a_{-4}^{j}$ or $w \leq_{R} b_{-5}^{j}$, we have that $A_{-4}^{j}$ is refuted at $a_{-4}^{j}$ and $B_{-4}^{j}$ is refuted at $b_{-4}^{j}$. Therefore, $w \not \forall A_{-4}^{j}$ if $w \leq_{R} a_{-4}^{j}$ and $w \not \forall B_{-4}^{j}$ if $w \leq_{R} b_{-4}^{j}$. 


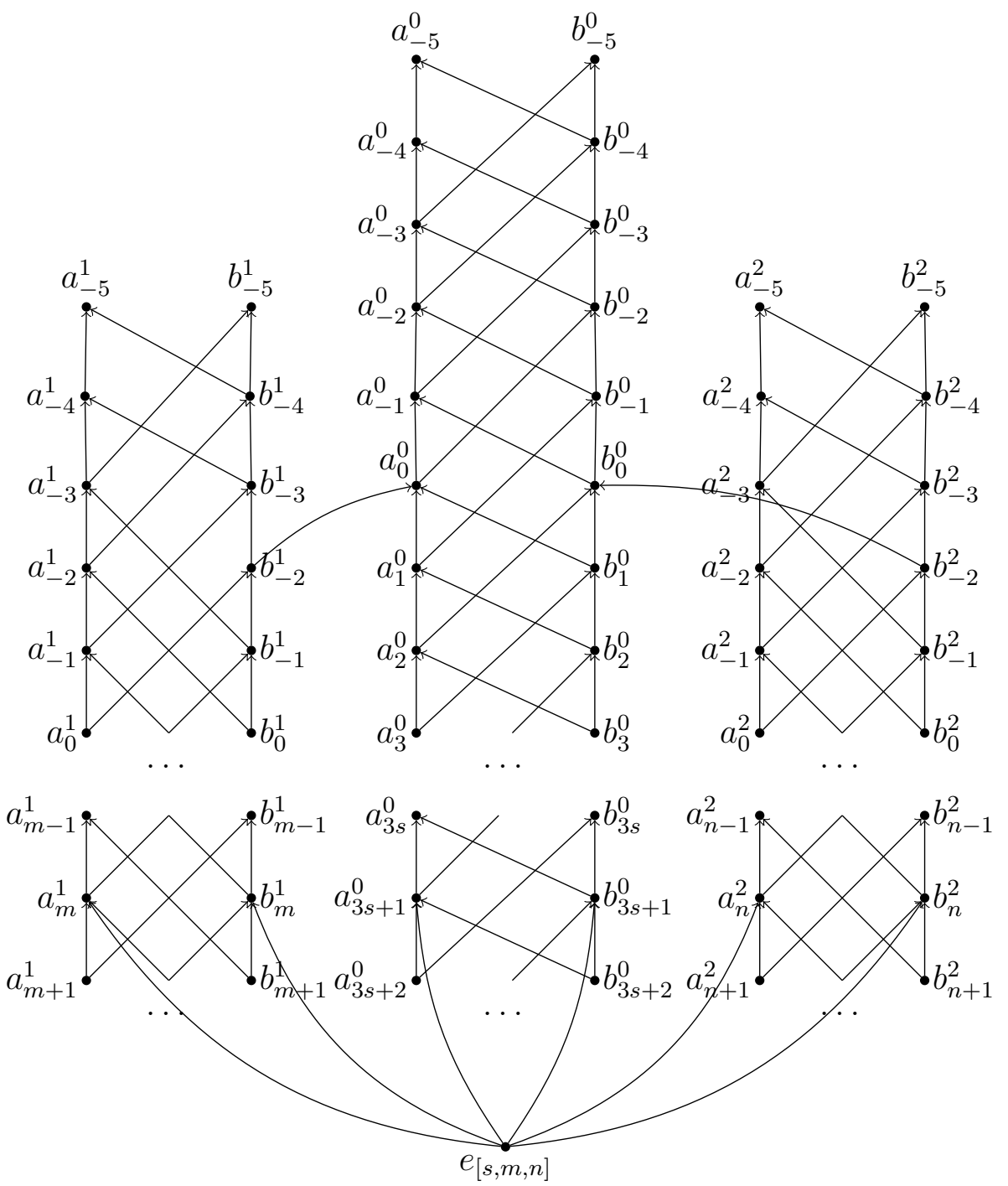

Figure 1: Kripke model $\mathfrak{M}$. 
If $w \not \models A_{-4}^{j}$, then there exists a point $w^{\prime} \geq_{R} w$ such that $w^{\prime} \models \neg \neg x_{j}$ and $w^{\prime} \not \models x_{j}$. By definition of the valuation $\mathfrak{V}$, we have either $w^{\prime} \leq_{R} a_{-4}^{j}$ or $w^{\prime} \leq_{R} b_{-5}^{j}$. Since $w^{\prime} \models \neg \neg x_{j}$, therefore for all point $w^{\prime \prime} \geq_{R} w^{\prime}$ there is a point $w^{\prime \prime \prime} \geq_{R} w^{\prime \prime}$ such that $w^{\prime \prime \prime} \models x_{j}$. It is clear that $w^{\prime} \underline{\leq}_{R} b_{-5}^{j}$. Hence, $w \leq_{R} a_{-4}^{j}$.

If $w \not \models B_{-4}^{j}$, then there exist points $w^{\prime} \geq_{R} w$ and $w^{\prime \prime} \geq_{R} w$ such that $w^{\prime} \models x_{j}$ and $w^{\prime \prime} \models \neg x_{j}$. By definition of the valuation $\mathfrak{V}$, we have $w^{\prime} \varliminf_{R} a_{-4}^{j}, w^{\prime} \varliminf_{R} b_{-5}^{j}$, and $w^{\prime \prime}=b_{-5}^{j}$. If $w^{\prime} \leq_{R} a_{-5}^{j^{\prime}}$ or $w^{\prime} \leq_{R} b_{-5}^{j^{\prime}}$ for some $j^{\prime} \in\{0,1,2\} \backslash\{j\}$, then $w \leq_{R} e_{[s, m, n]}$ for some $s, m, n \geq 0$ and therefore $w \leq_{R} b_{-4}^{j}$. Otherwise, $w^{\prime}=a_{-5}^{j}$. Note that there is a unique point $w^{\prime \prime \prime} \geq_{R} w$ such that $w^{\prime \prime \prime} \leq_{R} a_{-5}^{j}, w^{\prime \prime \prime} \leq_{R} b_{-5}^{j}$, and $w^{\prime \prime \prime} \models A_{-4}^{j}$. It is easily seen that $w^{\prime \prime \prime}=b_{-4}^{j}$ and therefore $w \leq_{R} b_{-4}^{j}$.

2) $i=-3$.

Note that $w \forall \forall A_{-3}^{j}$ if $w \leq_{R} a_{-4}^{j}, w \leq_{R} b_{-5}^{j}, w \not_{R} b_{-4}^{j}$ and $w \not \forall B_{-3}^{j}$ if $w \leq_{R} a_{-4}^{j}$, $w \leq_{R} b_{-4}^{j}, w \leq_{R} a_{-3}^{j}$. Since $a_{-3}^{j}$ and $b_{-3}^{j}$ are unique maximal points satisfying this condition, we have that $w \not \models A_{-3}^{j}$ if $w \leq_{R} a_{-3}^{j}$ and $w \not \forall B_{-3}^{j}$ if $w \leq_{R} b_{-3}^{j}$.

If $w \not \forall A_{-3}^{j}$, then there exists a point $w^{\prime} \geq_{R} w$ such that $w^{\prime} \not \models A_{-4}^{j}, \neg \neg x_{j}$ and $w^{\prime} \models B_{-4}^{j}$. So, $w^{\prime} \leq_{R} a_{-4}^{j}$ and $w^{\prime} \underline{\leq}_{R} b_{-4}^{j}$. Since $w^{\prime} \not \forall \neg \neg x_{j}$, there is a point $w^{\prime \prime} \geq_{R} w^{\prime}$ such that $w^{\prime \prime} \models \neg x_{j}$. It is clear that $w^{\prime \prime}=b_{-5}^{j}$. Evidently, the model $\mathfrak{M}$ contains only one point $a_{-3}^{j}$ satisfying the following condition: $w^{\prime} \leq_{R} a_{-4}^{j}, w^{\prime} \leq_{R} b_{-5}^{j}$ and $w^{\prime} \mathbb{L}_{R} b_{-4}^{j}$. Hence, $w \leq_{R} a_{-3}^{j}$.

If $w \not \models B_{-3}^{j}$, then there exists a point $w^{\prime} \geq_{R} w$ such that $w^{\prime} \not \models A_{-4}^{j}, B_{-4}^{j}$ and $w^{\prime} \models A_{-3}^{j}$. Then $w^{\prime} \leq_{R} a_{-4}^{j}, w^{\prime} \leq_{R} b_{-4}^{j}$ and $w^{\prime} \underline{\leq}_{R} a_{-3}^{j}$. Evidently, the model $\mathfrak{M}$ contains only one point $b_{-3}^{j}$ satisfying this condition. Therefore, $w \leq_{R} b_{-3}^{j}$.

3) $i=-2$ and $j \in\{1,2\}$.

We have that $w \not \models A_{-2}^{j}$ if $w \leq_{R} a_{-3}^{j}, w \leq_{R} b_{-4}^{j}, w{\leq_{R}}_{R} b_{-3}^{j}$ and $w \forall \forall B_{-2}^{j}$ if $w \leq_{R} c$, $w \leq_{R} b_{-3}^{j}, w \not_{R} a_{-2}^{j}$, where $c=a_{0}^{0}$ for $j=1$ and $c=b_{0}^{0}$ for $j=2$. Since $a_{-2}^{j}$ and $b_{-2}^{j}$ are unique maximal points satisfying this condition, we have that $w \forall A_{-2}^{j}$ if $w \leq_{R} a_{-2}^{j}$ and $w \not \models B_{-2}^{j}$ if $w \leq_{R} b_{-2}^{j}$.

If $w \not \models A_{-2}^{j}$, then there exists a point $w^{\prime} \geq_{R} w$ such that $w^{\prime} \not \models A_{-3}^{j}, B_{-4}^{j}$ and $w^{\prime} \models B_{-3}^{j}$. So, $w^{\prime} \leq_{R} a_{-3}^{j}, w^{\prime} \leq_{R} b_{-4}^{j}$, and $w^{\prime} \underline{\leq}_{R} b_{-3}^{j}$. It is clear that the model $\mathfrak{M}$ contains only one point $a_{-2}^{j}$ satisfying this condition. Hence, $w \leq_{R} a_{-2}^{j}$.

If $w \not \forall B_{-2}^{j}$, then there exists a point $w^{\prime} \geq_{R} w$ such that $w^{\prime} \not \models C_{j}, B_{-3}^{j}$ and $w^{\prime} \models A_{-3}^{j}$. Then $w^{\prime} \leq_{R} c, w^{\prime} \leq_{R} b_{-3}^{j}$ and $w^{\prime} \underline{\leq}_{R} a_{-3}^{j}$, where $c=a_{0}^{0}$ if $j=1$ and $c=b_{0}^{0}$ if $j=2$. Evidently, the model $\mathfrak{M}$ contains only one point $b_{-2}^{j}$ satisfying this condition. Therefore, $w \leq_{R} b_{-2}^{j}$.

4) $i=-1$ and $j \in\{1,2\}$. This case easily follows by analogy.

Induction step: assume that $i \geq-2$ if $j=0$ and $i \geq 0$ if $j \in\{1,2\}$. Without loss of generality, we can consider the case $j=1$. The cases $j=0$ and $j=2$ are proved by analogy.

By induction assumption, we have that $w \not \models A_{i}^{1}$ if $w \leq_{R} a_{0}^{0}, a_{i-1}^{1}, b_{i-2}^{1}, w \varliminf_{R} b_{0}^{0}, b_{i-1}^{1}$ and $w \not \models B_{i}^{1}$ if $w \leq_{R} a_{0}^{0}, a_{i-2}^{1}, b_{i-1}^{1}, w \varliminf_{R} b_{0}^{0}, a_{i-1}^{1}$. Since $a_{i}^{1}$ and $b_{i}^{1}$ are unique maximal points satisfying this condition, we have that $w \not \models A_{i}^{1}$ if $w \leq_{R} a_{i}^{1}$ and $w \not \models B_{i}^{1}$ if $w \leq_{R} b_{i}^{1}$.

If $w \forall \forall A_{i}^{1}$, then there exists a point $w^{\prime} \geq_{R} w$ such that $w^{\prime} \not C_{1}, A_{i-1}^{1}, B_{i-2}^{1}$ and $w^{\prime} \models C_{2}, B_{i-1}^{1}$. By induction hypothesis, we obtain that $w^{\prime} \leq_{R} a_{i-1}^{1}, w^{\prime} \leq_{R} b_{i-2}^{1}$, and $w^{\prime} \Varangle_{R}$ $b_{i-1}^{1}$. So, $w^{\prime}=a_{i}^{1}$ and $w \leq_{R} a_{i}^{1}$ by definition of the accessibility relation $R$. Analogously, if $w \not \models B_{i}^{1}$, then $w \leq_{R} b_{i}^{1}$. The lemma is proved. 
Lemma 3.4. Let $w$ be a world of $\mathfrak{M}$, then

$$
w \not=E_{s, m, n} \Longleftrightarrow w \leq_{R} e_{[s, m, n]}
$$

for all $s, m, n \geq 0$ such that $\alpha_{0} \stackrel{\mathcal{M}}{\Longleftrightarrow}[s, m, n]$.

The proof is trivial by definition of the accessibility relation $R$. Finally, we prove the key lemma of this section.

Lemma 3.5. If $(\mathfrak{F}, w) \forall \forall E_{s, m, n}$, then $e_{[s, m, n]} \in W$ and $w \leq_{R} e_{[s, m, n]}$ for all $s, m, n \geq 0$.

Proof. Let $\mathfrak{M}^{\prime}=\left\langle\mathfrak{F}, \mathfrak{V}^{\prime}\right\rangle$ be a Kripke model such that $\left(\mathfrak{M}^{\prime}, w\right) \not \forall E_{s, m, n}$. Since $w \forall \forall E_{s, m, n}$, there is a point $w^{\prime} \geq_{R} w$ such that the formulas $A_{3 s+1}^{0}, B_{3 s+1}^{0}, A_{m}^{1}, B_{m}^{1}, A_{n}^{2}, B_{n}^{2}$ are refuted at $w^{\prime}$, and the formulas $A_{3 s+2}^{0}, B_{3 s+2}^{0}, A_{m+1}^{1}, B_{m+1}^{1}, A_{n+1}^{2}, B_{n+1}^{2}$ are true at $w^{\prime}$.

Denote by $w_{s}^{a}$ and $w_{s}^{b}$ points of the frame $\mathfrak{F}$ such that

1. $w_{s}^{a} \geq_{R} w^{\prime}, w_{s}^{a} \models B_{3 s}^{0}$, and $w_{s}^{a} \not \models A_{3 s}^{0}, B_{3 s-1}^{0}$;

2. $w_{s}^{b} \geq_{R} w^{\prime}, w_{s}^{b} \models A_{3 s+1}^{0}$, and $w_{s}^{b} \not \models A_{3 s}^{0}, B_{3 s}^{0}$.

It is clear that these points exist.

If $w_{s}^{a}$ or $w_{s}^{b}$ are in $\left\{a_{n}^{0}, b_{n}^{0}\right\}$ for some $n \geq-5$, then $a_{-5}^{0} \in \mathfrak{V}^{\prime}(r), a_{-4}^{0}, b_{-5}^{0} \notin \mathfrak{V}^{\prime}(r)$. By analogy with Lemma 3.3, it is not hard to prove that $w_{s}^{a}=a_{3 s+1}^{0}, w_{s}^{b}=b_{3 s+1}^{0}$ by induction on $s \geq 0$.

Let $w_{s}^{a}$ and $w_{s}^{b}$ are not in $\left\{a_{n}^{0}, b_{n}^{0}\right\}$ for all $n \geq-5$. Then there are $n \geq-5$ and $j \in\{1,2\}$ such that $w_{s}^{a}$ or $w_{s}^{b}$ are in $\left\{a_{n}^{j}, b_{n}^{j}\right\}$. Evidently, either $a_{-5}^{0} \notin \mathfrak{V}^{\prime}(r), a_{-4}^{0} \in \mathfrak{V}^{\prime}(r)$, or $b_{-5}^{0} \notin \mathfrak{V}^{\prime}(r)$. We need to consider the following cases:

1. $a_{-5}^{j} \notin \mathfrak{V}^{\prime}(r)$. In this case, $A_{-4}^{0}$ is refuted at $a_{0}^{0}$ if $j=1$ and $b_{0}^{0}$ if $j=2$. Then it can easily be seen that $a_{-5}^{0}, b_{-5}^{0} \in \mathfrak{V}^{\prime}(r)$ and $a_{0}^{0} \notin \mathfrak{V}^{\prime}(r)$. If $b_{-5}^{j} \notin \mathfrak{V}^{\prime}(r)$, then $B_{-4}^{0}$ is true at $w_{s}^{a}, w_{s}^{b}$, which is impossible. If $b_{-5}^{j} \in \mathfrak{V}^{\prime}(r)$, then $B_{-4}^{0}$ is refuted at $a_{-3}^{j}, b_{-4}^{j}$ and therefore $A_{-3}^{0}$ is true at $w_{s}^{a}, w_{s}^{b}$, which is impossible. Hence $a_{-5}^{j} \in \mathfrak{V}^{\prime}(r)$.

2. $b_{-5}^{j} \in \mathfrak{V}^{\prime}(r)$. In this case, $B_{-4}^{0}$ is refuted at $b_{-2}^{j}$. Then $A_{-3}^{0}$ is true at $w_{s}^{a}, w_{s}^{b}$, which is impossible. Hence $b_{-5}^{j} \notin \mathfrak{V}^{\prime}(r)$.

3. $a_{-4}^{j} \in \mathfrak{V}^{\prime}(r)$. In this case, $A_{-4}^{0}$ is refuted at $a_{0}^{0}$ if $j=1$ and $b_{0}^{0}$ if $j=2$. As the above, we have that $a_{-5}^{0}, b_{-5}^{0} \in \mathfrak{V}^{\prime}(r)$ and $a_{0}^{0} \notin \mathfrak{V}^{\prime}(r)$. Then $B_{-4}^{0}$ is refuted at $a_{-3}^{j}, b_{-4}^{j}$ and therefore $A_{-3}^{0}$ is true at $w_{s}^{a}, w_{s}^{b}$, which is impossible. Hence $a_{-4}^{j} \notin \mathfrak{V}^{\prime}(r)$.

So, we have that $a_{-5}^{j} \in \mathfrak{V}^{\prime}(r)$ and $a_{-4}^{j}, b_{-5}^{j} \notin \mathfrak{V}^{\prime}(r)$. Then $A_{-4}^{0}$ is refuted at $a_{-4}^{j}$ and $B_{-4}^{0}$ is refuted at $b_{-4}^{j}$. It can easily be proved by induction on $s \geq 0$ that $w_{s}^{a}=a_{4 s+2}^{j}$ and $w_{s}^{b}=b_{4 s+3}^{j}$. Therefore, if $w_{s}^{a}=a_{4 s+2}^{j_{1}}$ and $w_{s}^{b}=b_{4 s+3}^{j_{2}}$ for some $j_{1}, j_{2} \in\{1,2\}$, then $w^{\prime} \leq_{R} a_{4 s+3}^{j_{2}}$ and therefore $A_{3 s+2}^{0}$ is refuted at $w^{\prime}$, which is impossible. Hence $w_{s}^{a}$ or $w_{s}^{b}$ are in $\left\{a_{n}^{0}, b_{n}^{0}\right\}$ for some $n \geq-5$ and therefore $a_{-5}^{0} \in \mathfrak{V}^{\prime}(r), a_{-4}^{0}, b_{-5}^{0} \notin \mathfrak{V}^{\prime}(r)$.

Since $C_{1}$ is refuted at $w_{1}$ if $w_{1} \leq_{R} a_{0}^{0}$ and $C_{2}$ is refuted at $w_{2}$ if $w_{2} \leq_{R} b_{0}^{0}$, we have that, for a given $i \geq 0$ and $j \in\{1,2\}$, the formulas $A_{i}^{j}, B_{i}^{j}$ are refuted at $a_{k}^{j}, b_{k}^{j}$ for some $k \geq 0$ and true at $a_{k}^{j^{\prime}}, b_{k}^{j^{\prime}}$ for all $k \geq 0, j^{\prime} \in\{0,1,2\} \backslash\{j\}$ by analogy with the above. Therefore, $a_{-5}^{1} \in \mathfrak{V}^{\prime}(p), a_{-4}^{1}, b_{-5}^{1} \notin \mathfrak{V}^{\prime}(p)$ and $a_{-5}^{2} \in \mathfrak{V}^{\prime}(q), a_{-4}^{2}, b_{-5}^{2} \notin \mathfrak{V}^{\prime}(q)$. 
Now if we recall the proof of Lemma 3.3, then we obtain that $w^{\prime} \leq_{R} a_{3 s+1}^{0}, w^{\prime} \leq_{R} b_{3 s+1}^{0}$, $w^{\prime} \leq_{R} a_{m}^{1}, w^{\prime} \leq_{R} b_{m}^{1}, w^{\prime} \leq_{R} a_{n}^{2}, w^{\prime} \leq_{R} b_{n}^{2}$ and $w^{\prime} \underline{\leq}_{R} a_{3 s+2}^{0}, w^{\prime} \leq_{R} b_{3 s+2}^{0}, w^{\prime} \underline{\leq}_{R} a_{m+1}^{1}$, $w^{\prime} \underline{\leq}_{R} b_{m+1}^{1}, w^{\prime} \underline{\leq}_{R} a_{n+1}^{2}, w^{\prime} \underline{\leq}_{R} b_{n+1}^{2}$. Evidently, the frame $\mathfrak{F}$ contains a unique maximal point $e_{[s, m, n]}$ satisfying this condition. Hence $e_{[s, m, n]} \in W$ and $w \leq_{R} w^{\prime} \leq_{R} e_{[s, m, n]}$. The lemma is proved.

\subsection{Key formulas}

In this section, we consider the key formulas depending on variables $p, q, r$. First, let us define the following formulas $F_{k}=F_{k}[p, q, x, y]$ and $G_{k}=G_{k}[p, q, x, y]$ in variables $p, q, x$ and $y$ :

$$
\begin{aligned}
& F_{0}=p, \\
& G_{0}=q, \\
& F_{1}=y \wedge q \rightarrow x \vee p, \\
& G_{1}=y \wedge p \rightarrow x \vee q, \text { and } \\
& F_{k}=y \wedge G_{k-1} \rightarrow x \vee F_{k-1} \vee G_{k-2}, \\
& G_{k}=y \wedge F_{k-1} \rightarrow x \vee G_{k-1} \vee F_{k-2}, \text { for all } k \geq 2 .
\end{aligned}
$$

Now we introduce the following key formulas:

$$
\begin{aligned}
F_{k}^{1}[p, q] & =F_{k}\left[p, q, C_{1}, C_{2}\right] \\
G_{k}^{1}[p, q] & =G_{k}\left[p, q, C_{1}, C_{2}\right] \\
F_{k}^{2}[p, q] & =F_{k}\left[p, q, C_{2}, C_{1}\right] \\
G_{k}^{2}[p, q] & =G_{k}\left[p, q, C_{2}, C_{1}\right]
\end{aligned}
$$

Note that the formulas $F_{k}^{m}$ and $G_{k}^{m}$ are depending on three variables $p, q$, and $r$, for all $k \geq 0$ and $m \in\{1,2\}$.

Besides, we define the following auxiliary formulas:

$$
\begin{aligned}
& P_{i, j}=\left(C_{2} \rightarrow C_{1} \vee A_{i}^{1} \vee B_{i-1}^{1}\right) \wedge\left(C_{1} \rightarrow C_{2} \vee A_{i}^{2} \vee B_{i-1}^{2}\right), \\
& Q_{i, j}=\left(C_{2} \rightarrow C_{1} \vee A_{i-1}^{1} \vee B_{i}^{1}\right) \wedge\left(C_{1} \rightarrow C_{2} \vee A_{i-1}^{2} \vee B_{i}^{2}\right),
\end{aligned}
$$

for all $i, j \geq-1$. The following lemma is describing the basic properties of the key formulas.

Lemma 3.6. For all $i, j \geq-1, k \geq 1$ and $m \in\{1,2\}$,

$$
\begin{aligned}
& \text { Int } \vdash F_{k}^{m}\left[P_{i, j}, Q_{i, j}\right] \leftrightarrow A_{n+k}^{m}, \\
& \text { Int } \vdash G_{k}^{m}\left[P_{i, j}, Q_{i, j}\right] \leftrightarrow B_{n+k}^{m},
\end{aligned}
$$

where

$$
n= \begin{cases}i, & m=1 \\ j, & m=2\end{cases}
$$

Proof. By induction on $k \geq 1$. Without loss of generality, we can assume that $m=1$. The basis of induction consists of two cases: $k=1$ and $k=2$.

Induction base: $k=1$. In this case we have

$$
\begin{aligned}
& F_{1}^{1}\left[P_{i, j}, Q_{i, j}\right]=C_{2} \wedge Q_{i, j} \rightarrow C_{1} \vee P_{i, j} \\
& G_{1}^{1}\left[P_{i, j}, Q_{i, j}\right]=C_{2} \wedge P_{i, j} \rightarrow C_{1} \vee Q_{i, j}
\end{aligned}
$$


It can easily be checked that the following derivations holds in Int:

$$
\begin{aligned}
& \text { Int } \vdash C_{2} \wedge B_{i}^{1} \rightarrow C_{2} \wedge Q_{i, j}, \quad \text { Int } \vdash C_{1} \vee P_{i, j} \rightarrow\left(C_{2} \rightarrow C_{1} \vee A_{i}^{1} \vee B_{i-1}^{1}\right), \\
& \text { Int } \vdash C_{2} \wedge A_{i}^{1} \rightarrow C_{2} \wedge P_{i, j}, \quad \text { Int } \vdash C_{1} \vee Q_{i, j} \rightarrow\left(C_{2} \rightarrow C_{1} \vee A_{i-1}^{1} \vee B_{i}^{1}\right)
\end{aligned}
$$

Hence,

$$
\begin{aligned}
& \text { Int } \vdash F_{1}^{1}\left[P_{i, j}, Q_{i, j}\right] \rightarrow A_{i+1}^{1}, \\
& \text { Int } \vdash G_{1}^{1}\left[P_{i, j}, Q_{i, j}\right] \rightarrow B_{i+1}^{1} .
\end{aligned}
$$

Conversely, since the formulas $A_{i-1}^{1} \rightarrow A_{i}^{1}$ and $B_{i-1}^{1} \rightarrow B_{i}^{1}$ are derivable from Int, we have

$$
\begin{aligned}
& \text { Int, } A_{i+1}^{1} \vdash C_{1} \vee A_{i-1}^{1} \vee B_{i}^{1} \rightarrow\left(C_{2} \rightarrow C_{1} \vee A_{i}^{1} \vee B_{i-1}^{1}\right), \\
& \text { Int, } B_{i+1}^{1} \vdash C_{1} \vee A_{i}^{1} \vee B_{i-1}^{1} \rightarrow\left(C_{2} \rightarrow C_{1} \vee A_{i-1}^{1} \vee B_{i}^{1}\right)
\end{aligned}
$$

and therefore the following derivations holds in Int:

$$
\begin{array}{ll}
\text { Int, } A_{i+1}^{1} & \vdash C_{2} \wedge\left(C_{2} \rightarrow C_{1} \vee A_{i-1}^{1} \vee B_{i}^{1}\right) \rightarrow P_{i, j}, \\
\text { Int, } B_{i+1}^{1} \vdash C_{2} \wedge\left(C_{2} \rightarrow C_{1} \vee A_{i}^{1} \vee B_{i-1}^{1}\right) \rightarrow Q_{i, j}
\end{array}
$$

Hence,

$$
\begin{aligned}
& \text { Int } \vdash A_{i+1}^{1} \rightarrow F_{1}^{1}\left[P_{i, j}, Q_{i, j}\right], \\
& \text { Int } \vdash \quad B_{i+1}^{1} \rightarrow G_{1}^{1}\left[P_{i, j}, Q_{i, j}\right] .
\end{aligned}
$$

Induction base: $k=2$. In this case we have

$$
\begin{aligned}
& \text { Int } \vdash F_{2}^{1}\left[P_{i, j}, Q_{i, j}\right] \leftrightarrow\left(C_{2} \wedge B_{i+1}^{1} \rightarrow C_{1} \vee A_{i+1}^{1} \vee Q_{i, j}\right), \\
& \text { Int } \vdash G_{2}^{1}\left[P_{i, j}, Q_{i, j}\right] \leftrightarrow\left(C_{2} \wedge A_{i+1}^{1} \rightarrow C_{1} \vee B_{i+1}^{1} \vee P_{i, j}\right) .
\end{aligned}
$$

Furthermore, it follows easily that:

$$
\begin{aligned}
& \text { Int } \vdash C_{2} \wedge B_{i}^{1} \rightarrow Q_{i, j}, \quad \text { Int } \vdash Q_{i, j} \rightarrow\left(C_{2} \rightarrow C_{1} \vee A_{i+1}^{1} \vee B_{i}^{1}\right), \\
& \text { Int } \vdash C_{2} \wedge A_{i}^{1} \rightarrow P_{i, j}, \quad \text { Int } \vdash P_{i, j} \rightarrow\left(C_{2} \rightarrow C_{1} \vee A_{i}^{1} \vee B_{i+1}^{1}\right)
\end{aligned}
$$

Hence,

$$
\begin{aligned}
& \text { Int } \vdash \quad F_{2}^{1}\left[P_{i, j}, Q_{i, j}\right] \leftrightarrow A_{i+2}^{1}, \\
& \text { Int } \vdash G_{2}^{1}\left[P_{i, j}, Q_{i, j}\right] \leftrightarrow B_{i+2}^{1} .
\end{aligned}
$$

Induction step is straightforward and left to the reader. The lemma is proved.

\subsection{Encoding of the Minsky machine}

Now we encode instructions of the Minsky machine $\mathcal{M}$ as superintuitionistic formulas such that derivations from Int and these formulas are simulate transformations of $\mathcal{M}$.

First, let us define the following formulas containing only tree variables $p, q, r$ :

$$
\begin{aligned}
\hat{E}_{s, i, j} & =A_{3 s+2}^{0} \wedge B_{3 s+2}^{0} \wedge F_{i+1}^{1} \wedge G_{i+1}^{1} \wedge F_{j+1}^{2} \wedge G_{j+1}^{2} \rightarrow \\
& \rightarrow A_{3 s+1}^{0} \vee B_{3 s+1}^{0} \vee F_{i}^{1} \vee G_{i}^{1} \vee F_{j}^{2} \vee G_{j}^{2}, \\
\hat{E}_{s, 0, *} & =A_{3 s+2}^{0} \wedge B_{3 s+2}^{0} \wedge A_{1}^{1} \wedge B_{1}^{1} \rightarrow A_{3 s+1}^{0} \vee B_{3 s+1}^{0} \vee A_{0}^{1} \vee B_{0}^{1} \vee q, \\
\hat{E}_{s, *, 0} & =A_{3 s+2}^{0} \wedge B_{3 s+2}^{0} \wedge A_{1}^{2} \wedge B_{1}^{2} \rightarrow A_{3 s+1}^{0} \vee B_{3 s+1}^{0} \vee p \vee A_{0}^{2} \vee B_{0}^{2}, \\
\hat{E}_{s, 0,0} & =E_{s, 0,0},
\end{aligned}
$$

where $s \geq 0, i, j \geq 1$. By Lemma 3.6, we have the following evident lemma. 
Lemma 3.7. For all $s, m, n \geq 0$,

$$
\boldsymbol{I n t} \vdash E_{s, m, n} \leftrightarrow \begin{cases}\hat{E}_{s, i, j}\left[P_{m-i, n-j}, Q_{m-i, n-j}\right], & 1 \leq i \leq m+1, \\ A_{n+1}^{2} \wedge B_{n+1}^{2} \rightarrow \hat{E}_{s, 0, *}\left[p, A_{n}^{2} \vee B_{n}^{2}\right], & 1 \leq j \leq n+1 ; \\ A_{m+1}^{1} \wedge B_{m+1}^{1} \rightarrow \hat{E}_{s, *, 0}\left[A_{m}^{1} \vee B_{m}^{1}, q\right], & m \geq 1, n=0 ; \\ \hat{E}_{s, 0,0}, & m=0, n=0 .\end{cases}
$$

Let

$$
\varphi(x)= \begin{cases}x-1, & x \geq 1 \\ 0, & x=0 \\ 0, & x=*\end{cases}
$$

Now we prove that if the Kripke frame $\mathfrak{F}$ refutes $\hat{E}_{s, i, j}$ then it refutes $\hat{E}_{s, i, j}$ at a point $e_{[s, m, n]}$ for some $m \geq \varphi(i), n \geq \varphi(j)$ such that $\alpha_{0} \stackrel{\mathcal{M}}{\Longleftrightarrow}[s, m, n]$.

Lemma 3.8. If $(\mathfrak{F}, w) \not \hat{E}_{s, i, j}$, then $w \leq_{R} e_{[s, m, n]}$ for some $m \geq \varphi(i), n \geq \varphi(j)$ such that $\alpha_{0} \stackrel{\mathcal{M}}{\Longrightarrow}[s, m, n]$.

Proof. Let $\mathfrak{M}^{\prime}=\left\langle\mathfrak{F}, \mathfrak{V}^{\prime}\right\rangle$ be a Kripke model such that $\left(\mathfrak{M}^{\prime}, w\right) \not \forall \hat{E}_{s, i, j}$. Since $w \not \models \hat{E}_{s, i, j}$, there is a point $w^{\prime} \geq_{R} w$ such that the formulas $A_{3 s+1}^{0}$ and $B_{3 s+1}^{0}$ are refuted at $w^{\prime}$, and the formulas $A_{3 s+2}^{0}$ and $B_{3 s+2}^{0}$ are true at $w^{\prime}$. By the proof of Lemma 3.5, we have that $w \leq_{R} w^{\prime} \leq_{R} e_{[s, m, n]}$ for some $m, n \geq 0$ such that $\alpha_{0} \stackrel{\mathcal{M}}{\Longrightarrow}[s, m, n]$. It is clear that $m=0$ if $i=0$ and $n=0$ if $j=0$. Hence, in order to prove the lemma it is sufficient to show that $m \geq i-1, n \geq j-1$ for some $i \geq 1, j \geq 1$.

If $i \geq 1$, then the formulas $F_{i}^{1}, G_{i}^{1}$ are refuted at $w^{\prime}$ and the formulas $F_{i+1}^{1}, G_{i+1}^{1}$ are true at $w^{\prime}$. Now we prove that if $F_{k}^{1}$ is refuted at a point $f_{k}^{1}$ and $G_{k}^{1}$ is refuted at a point $g_{k}^{1}$, then $f_{k}^{1} \leq_{R} c_{k+l-1}^{1}$ and $g_{k}^{1} \leq_{R} d_{k+l-1}^{1}$ for some $l \geq 0$ and $\{c, d\}=\{a, b\}$. By induction on $k \geq 1$.

Induction base: $k=1$. In this case, there are points $w_{f} \geq_{R} f_{1}^{1}$ and $w_{g} \geq_{R} g_{1}^{1}$ such that

1. $C_{1}$ is refuted at $w_{f}, w_{g}$, then the Kripke frame $\mathfrak{F}$ contains pathes of length 5 from $w_{f}$, $w_{g}$ to maximal points and therefore $w_{f} \leq_{R} c_{0}^{j_{1}}$ and $w_{g} \leq_{R} d_{0}^{j_{2}}$ for some $j_{1}, j_{2} \in\{0,1,2\}$ and $c, d \in\{a, b\}$;

2. $C_{2}$ is true at $w_{f}, w_{g}$, therefore $w_{f} \not_{R} b_{-1}^{0}, w_{g} \leq_{R} b_{-1}^{0}$ by the proof of Lemma 3.5,

3. $w_{f} \in \mathfrak{V}^{\prime}(q) \backslash \mathfrak{V}^{\prime}(p)$ and $w_{g} \in \mathfrak{V}^{\prime}(p) \backslash \mathfrak{V}^{\prime}(q)$, therefore $w_{f}, w_{g}$ are incomparable points.

Thus, $w_{f}=c_{i^{\prime}}^{1}$ and $w_{g}=d_{j^{\prime}}^{1}$ for some $i^{\prime}, j^{\prime} \geq 0$ such that $\left|i^{\prime}-j^{\prime}\right|<2$, and $\{c, d\}=\{a, b\}$.

Induction base: $k=2$. In this case, there are points $w_{f} \geq_{R} f_{2}^{1}$ and $w_{g} \geq_{R} g_{2}^{1}$ such that

1. $F_{1}^{1}$ is refuted at $w_{f}$ and $G_{1}^{1}$ is refuted at $w_{g}$, therefore $w_{f} \leq_{R} c_{i^{\prime}}^{1}, w_{g} \leq_{R} d_{j^{\prime}}^{1}$;

2. $F_{1}^{1}$ is true at $w_{g}$ and $G_{1}^{1}$ is true at $w_{f}$, therefore $w_{f} \not_{R} d_{j^{\prime}}^{1}, w_{g} \bigsqcup_{R} c_{i^{\prime}}^{1}$;

3. $w_{f}, w_{g} \notin \mathfrak{V}^{\prime}(p) \cup \mathfrak{V}^{\prime}(q)$, therefore $w_{f} \neq c_{i^{\prime}}^{1}, w_{g} \neq d_{j^{\prime}}^{1}$. 
Thus, $w_{f}=c_{i^{\prime \prime}}^{1}, w_{g}=d_{j^{\prime \prime}}^{1}$ and $\left(w_{f}, d_{j^{\prime}}^{1}\right),\left(c_{i^{\prime}}^{1}, w_{g}\right)$ and $\left(w_{f}, w_{g}\right)$ are pairs of incomparable points. So, we have

$$
\begin{aligned}
& i^{\prime}<i^{\prime \prime}<j^{\prime}+2, \\
& j^{\prime}<j^{\prime \prime}<i^{\prime}+2 .
\end{aligned}
$$

Since $\left|i^{\prime}-j^{\prime}\right|<2$, it can easily be checked that $i^{\prime}=j^{\prime}=l$ and $i^{\prime \prime}=j^{\prime \prime}=l+1$ for some $l \geq 0$.

Induction step: $k>2$. Let the induction assumption be satisfied for all $2 \leq k^{\prime}<k$, then there are points $w_{f} \geq_{R} f_{k}^{1}$ and $w_{g} \geq_{R} g_{k}^{1}$ such that

1. $F_{k-1}^{1}, G_{k-2}^{1}$ are refuted at $w_{f}$, therefore $w_{f} \leq_{R} c_{k+l-2}^{1}, w_{f} \leq_{R} d_{k+l-3}^{1}$;

2. $G_{k-1}^{1}, F_{k-2}^{1}$ are refuted at $w_{g}$, therefore $w_{g} \leq_{R} d_{k+l-2}^{1}, w_{g} \leq_{R} c_{k+l-3}^{1}$;

3. $G_{k-1}^{1}$ is true at $w_{f}$ and $F_{k-1}^{1}$ is true at $w_{g}$, therefore $w_{f} \Varangle_{R} d_{k+l-2}^{1}$ and $w_{g} \Varangle_{R} c_{k+l-2}^{1}$. Thus, $w_{f}=c_{k+l-1}^{1}$ and $w_{g}=d_{k+l-1}^{1}$.

Since $F_{i}^{1}, G_{i}^{1}$ are refuted at $w^{\prime}$ and the formulas $F_{i+1}^{1}, G_{i+1}^{1}$ are true at $w^{\prime}$, we have $w^{\prime} \leq_{R} c_{i+l-1}^{1}, w^{\prime} \leq_{R} d_{i+l-1}^{1}$ and $w^{\prime} \not_{R} c_{i+l}^{1}, w^{\prime} \not_{R} d_{i+l}^{1}$. Therefore, $m=i+l-1 \geq i-1$.

If $j \geq 1$, then the proof are similar. Hence, $n \geq j-1$. The lemma is proved.

Next, we define the formula $A x(I)$ simulating the instruction $I$ of the Minsky machine $\mathcal{M}$ :

1. If $I$ is an instruction of the form $s \mapsto\langle t, 1,0\rangle$, then $A x(I)$ is the following formula

$$
\hat{E}_{t, 2,1} \rightarrow \hat{E}_{s, 1,1}
$$

2. If $I$ is $s \mapsto\langle t, 0,1\rangle$, then $A x(I)$ is

$$
\hat{E}_{t, 1,2} \rightarrow \hat{E}_{s, 1,1}
$$

3. If $I$ is $s \mapsto\langle t,-1,0\rangle /\langle u, 0,0\rangle$, then $A x(I)$ is

$$
\left(\hat{E}_{t, 1,1} \rightarrow \hat{E}_{s, 2,1}\right) \wedge\left(\hat{E}_{u, 0, *} \rightarrow \hat{E}_{s, 0, *}\right)
$$

4. If $I$ is $s \mapsto\langle t, 0,-1\rangle /\langle u, 0,0\rangle$, then $A x(I)$ is

$$
\left(\hat{E}_{t, 1,1} \rightarrow \hat{E}_{s, 1,2}\right) \wedge\left(\hat{E}_{u, *, 0} \rightarrow \hat{E}_{s, *, 0}\right)
$$

and the formula $A x(\mathcal{M})$ simulating the behavior of $\mathcal{M}$ itself:

$$
A x(\mathcal{M})=\bigwedge_{I \in \mathcal{M}} A x(I)
$$

Lemma 3.9. $\mathfrak{F} \models A x(\mathcal{M})$.

Proof. In order to prove the lemma it is sufficient to show that

$$
\mathfrak{F} \models A x(I)
$$

for each instruction $I$. We need to consider the following 4 cases. 
Case 1: $I$ is an instruction of the form $s \mapsto\langle t, 1,0\rangle$, i.e.,

$$
A x(I)=\hat{E}_{t, 2,1} \rightarrow \hat{E}_{s, 1,1} \text {. }
$$

If $(\mathfrak{F}, w) \not \models A x(I)$, then there is a Kripke model $\mathfrak{M}^{\prime}=\left\langle\mathfrak{F}, \mathfrak{V}^{\prime}\right\rangle$ such that $\left(\mathfrak{M}^{\prime}, w\right) \models \hat{E}_{t, 2,1}$ and $\left(\mathfrak{M}^{\prime}, w\right) \forall \hat{E}_{s, 1,1}$. By Lemma 3.8, $w \leq_{R} e_{[s, m, n]}$ for some $m \geq 0$ and $n \geq 0$ such that $\alpha_{0} \stackrel{\mathcal{M}}{\Longleftrightarrow}[s, m, n]$. If we recall the proofs of Lemmas 3.5 and 3.8, we obtain that the following statements hold in $\mathfrak{M}^{\prime}$

1. $A_{3 t+1}^{0}, B_{3 t+1}^{0}$ are refuted at $a_{3 t+1}^{0}, b_{3 t+1}^{0}$ and $A_{3 t+2}^{0}, B_{3 t+2}^{0}$ are true at them;

2. $F_{2}^{1}, G_{2}^{1}$ are refuted at $c_{m+1}^{1}, d_{m+1}^{1}$ and $F_{3}^{1}, G_{3}^{1}$ are true at them, where $\{c, d\}=\{a, b\}$;

3. $F_{1}^{2}, G_{1}^{2}$ are refuted at $c_{n}^{2}, d_{n}^{2}$ and $F_{2}^{2}, G_{2}^{2}$ are true at them, where $\{c, d\}=\{a, b\}$.

Since

$$
\langle s, m, n\rangle \stackrel{\mathcal{M}}{\longmapsto}\langle t, m+1, n\rangle,
$$

we have that $e_{[t, m+1, n]} \in W$ and $e_{[s, m, n]} \leq_{R} e_{[t, m+1, n]}$. Hence $\hat{E}_{t, 2,1}$ is refuted at $w$, which contradicts to that $\left(\mathfrak{M}^{\prime}, w\right) \models \hat{E}_{t, 2,1}$. Therefore, $(\mathfrak{F}, w) \models A x(I)$.

Case 2: $I$ is an instruction of the form $s \mapsto\langle t, 0,1\rangle$. The proof is analogous.

Case 3: $I$ is an instruction of the form $s \mapsto\langle t,-1,0\rangle /\langle u, 0,0\rangle$, i.e.,

$$
\left(\hat{E}_{t, 1,1} \rightarrow \hat{E}_{s, 2,1}\right) \wedge\left(\hat{E}_{u, 0, *} \rightarrow \hat{E}_{s, 0, *}\right) .
$$

Let $(\mathfrak{F}, w) \forall \neq A x(I)$. Then there is a Kripke model $\mathfrak{M}^{\prime}=\left\langle\mathfrak{F}, \mathfrak{V}^{\prime}\right\rangle$ such that

$$
\left(\mathfrak{M}^{\prime}, w\right) \not \models\left(\hat{E}_{t, 1,1} \rightarrow \hat{E}_{s, 2,1}\right),\left(\hat{E}_{u, 0, *} \rightarrow \hat{E}_{s, 0, *}\right) .
$$

It is clear that if $\left(\mathfrak{M}^{\prime}, w\right) \not \forall \hat{E}_{s, 2,1}$, then $\left(\mathfrak{M}^{\prime}, w\right) \not \forall \hat{E}_{t, 1,1}$. Let $\left(\mathfrak{M}^{\prime}, w\right) \not \forall \hat{E}_{s, 0, *}$ for some point

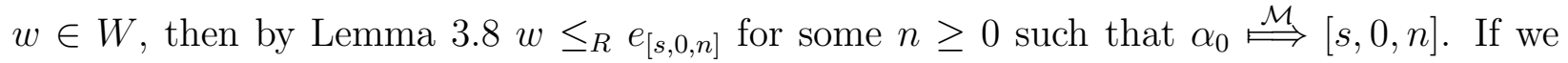
recall the proofs of Lemmas 3.5 and 3.8 again, we obtain that

1. $A_{3 u+1}^{0}, B_{3 u+1}^{0}$ are refuted at $a_{3 u+1}^{0}, b_{3 u+1}^{0}$ and $A_{3 u+2}^{0}, B_{3 u+2}^{0}$ are true at them;

2. $A_{0}^{1}, B_{0}^{1}$ are refuted at $a_{0}^{1}, b_{0}^{1}$ and $A_{1}^{1}, B_{1}^{1}$ are true at them.

Since

$$
\langle s, 0, n\rangle \stackrel{\mathcal{M}}{\longmapsto}\langle u, 0, n\rangle,
$$

we have that $e_{[u, 0, n]} \in W$ and $e_{[s, 0, n]} \leq_{R} e_{[u, 0, n]}$. Hence $\left(\mathfrak{M}^{\prime}, w\right) \not \hat{E}_{u, 0, *}$ and therefore $(\mathfrak{F}, w) \models A x(I)$.

Case 4: $I$ is an instruction of the form $s \mapsto\langle t, 0,-1\rangle /\langle u, 0,0\rangle$. The proof is similar. Thus, $\mathfrak{F} \models A x(I)$ for each instruction $I \in \mathcal{M}$. The lemma is proved. 


\subsection{Reduction of configuration problem}

In this section we formally reduce the configuration problem of the Minsky machine $\mathcal{M}$ to the derivation problem of the superintuitionistic propositional calculus Int $+A x(\mathcal{M})$.

Lemma 3.10. Int $+A x(\mathcal{M}) \vdash E_{t, k, l} \rightarrow E_{s_{0}, m_{0}, n_{0}}$ iff $\left\langle s_{0}, m_{0}, n_{0}\right\rangle \stackrel{\mathcal{M}}{\Longleftrightarrow}\langle t, k, l\rangle$.

Proof. If Int $+A x(\mathcal{M}) \vdash E_{t, k, l} \rightarrow E_{s_{0}, m_{0}, n_{0}}$, then

$$
\mathfrak{F} \models E_{t, k, l} \rightarrow E_{s_{0}, m_{0}, n_{0}}
$$

by Lemma 3.9. If we recall that $E_{s_{0}, m_{0}, n_{0}}$ is refuted at $e_{\left[s_{0}, m_{0}, n_{0}\right]}$, then we obtain that $E_{t, k, l}$ is also refuted at $e_{\left[s_{0}, m_{0}, n_{0}\right]}$. By Lemma 3.5, $e_{[t, k, l]} \in W$ and

$$
e_{\left[s_{0}, m_{0}, n_{0}\right]} \leq_{R} e_{[t, k, l]}
$$

Therefore, $\left\langle s_{0}, m_{0}, n_{0}\right\rangle \stackrel{\mathcal{M}}{\Longleftrightarrow}\langle t, k, l\rangle$ by definition of Kripke frame $\mathfrak{F}$.

Conversely, if $\left\langle s_{0}, m_{0}, n_{0}\right\rangle \stackrel{\mathcal{M}}{\Longleftrightarrow}\langle t, k, l\rangle$, then there exists a finite sequence $\left\langle s_{i}, m_{i}, n_{i}\right\rangle$, $0 \leq i \leq \mu$, such that $\left\langle s_{\mu}, m_{\mu}, n_{\mu}\right\rangle=\langle t, k, l\rangle$ and

$$
\left\langle s_{i}, m_{i}, n_{i}\right\rangle \stackrel{\mathcal{M}}{\longmapsto}\left\langle s_{i+1}, m_{i+1}, n_{i+1}\right\rangle
$$

for all $i, 0 \leq i<\mu$. Let $\left\langle s_{i+1}, m_{i+1}, n_{i+1}\right\rangle$ be a result of applying of an instruction $I \in \mathcal{M}$. We need to consider the following 4 cases.

Case 1: $I$ is an instruction of the form $s \mapsto\langle t, 1,0\rangle$. Then $m_{i+1}=m_{i}+1$ and $n_{i+1}=n_{i}$. By Lemma 3.7, we have

$$
\begin{aligned}
& \text { Int } \vdash E_{s_{i+1}, m_{i+1}, n_{i+1}} \leftrightarrow \hat{E}_{s_{i+1}, 2,1}\left[P_{m_{i}-1, n_{i}-1}, Q_{m_{i}-1, n_{i}-1}\right], \\
& \text { Int } \vdash E_{s_{i}, m_{i}, n_{i}} \leftrightarrow \hat{E}_{s_{i}, 1,1}\left[P_{m_{i}-1, n_{i}-1}, Q_{m_{i}-1, n_{i}-1}\right] .
\end{aligned}
$$

Therefore Int $+A x(\mathcal{M}) \vdash E_{s_{i+1}, m_{i+1}, n_{i+1}} \rightarrow E_{s_{i}, m_{i}, n_{i}}$

Case 2: $I$ is an instruction of the form $s \mapsto\langle t, 0,1\rangle$. The proof is analogous.

Case 3: $I$ is an instruction of the form $s \mapsto\langle t,-1,0\rangle /\langle u, 0,0\rangle$. If $m_{i+1}=m_{i}-1 \geq 0$ and $n_{i+1}=n_{i}$. By Lemma 3.7, we have

$$
\begin{aligned}
& \text { Int } \vdash E_{s_{i+1}, m_{i+1}, n_{i+1}} \leftrightarrow \hat{E}_{s_{i+1}, 1,1}\left[P_{m_{i}-2, n_{i}-1}, Q_{m_{i}-2, n_{i}-1}\right], \\
& \text { Int } \vdash \quad E_{s_{i}, m_{i}, n_{i}} \leftrightarrow \hat{E}_{s_{i}, 2,1}\left[P_{m_{i}-2, n_{i}-1}, Q_{m_{i}-2, n_{i}-1}\right] .
\end{aligned}
$$

If $m_{i+1}=m_{i}=0$ and $n_{i+1}=n_{i}$. By Lemma 3.7, we have

$$
\begin{aligned}
& \text { Int } \vdash E_{s_{i+1}, m_{i+1}, n_{i+1}} \leftrightarrow\left(A_{n_{i}+1}^{2} \wedge B_{n_{i}+1}^{2} \rightarrow \hat{E}_{s_{i+1}, 0, *}\left[p, A_{n_{i}}^{2} \vee B_{n_{i}}^{2}\right]\right), \\
& \text { Int } \vdash E_{s_{i}, m_{i}, n_{i}} \leftrightarrow\left(A_{n_{i}+1}^{2} \wedge B_{n_{i}+1}^{2} \rightarrow \hat{E}_{s_{i}, 0, *}\left[p, A_{n_{i}}^{2} \vee B_{n_{i}}^{2}\right]\right)
\end{aligned}
$$

Therefore Int $+A x(\mathcal{M}) \vdash E_{s_{i+1}, m_{i+1}, n_{i+1}} \rightarrow E_{s_{i}, m_{i}, n_{i}}$.

Case 4: $I$ is an instruction of the form $s \mapsto\langle t, 0,-1\rangle /\langle u, 0,0\rangle$. The proof is similar.

Thus, Int $+A x(\mathcal{M}) \vdash E_{s_{i+1}, m_{i+1}, n_{i+1}} \rightarrow E_{s_{i}, m_{i}, n_{i}}$ for all $i, 0 \leq i<\mu$. The lemma is proved.

Since the configuration problem for the Minsky machine $\mathcal{M}$ and the initial configuration $\left\langle s_{0}, m_{0}, n_{0}\right\rangle$ is undecidable by Theorem 3.2 , we have that the derivation problem for the superintuitionistic propositional calculus Int $+A x(\mathcal{M})$ is also undecidable. This completes the proof of Theorem 3.1 . 


\section{Conclusion and further research}

In this paper, we established that there is an undecidable superintuitionistic propositional calculus using axioms in only 3 variables. Since there are no undecidable superintuitionistic propositional calculi with axioms containing less than 3 variables, therefore a natural and interesting question is there an intuitionistic propositional formula $A$ containing less than 3 variables for which the superintuitionistic propositional calculus Int $+A$ is undecidable. In this respect, we note that every intermediate logic axiomatised by a 1-variable formula has the finite model property [18] and therefore decidable, but there exists an intermediate logic axiomatised by a 2-variable formula, which is Kripke incomplete [14].

\section{References}

[1] Bokov G. V. Completeness problem in the propositional calculus. // Intelligent Systems, vol. 13, no. 1-4, p. 165-182, 2009. (Russian).

[2] Bokov G. V. Undecidability of the problem of recognizing axiomatizations for propositional calculi with implication. // Logic Journal of the IGPL, vol. 23, no. 2, p. 341-353, 2015.

[3] Bokov G. V. Undecidable problems for propositional calculi with implication. // Logic Journal of the IGP, 2015. (Received June 4, 2015).

[4] Chagrov A. Undecidable properties of superintuitionistic logics. // Mathematical Problems of Cybernetics, vol. 5, p. 67-108, 1994. (Russian).

[5] Chagrov A., Zakharyaschev M. Modal Logic. — Clarendon Press, 1997.

[6] Gladstone M. D. On the number of variables in the axioms,. // Notre Dame Journal of Formal Logic, vol. 11, p. 1-15, 1970.

[7] Gladstone M. D. The decidability of one-variable propositional calculi. // Notre Dame Journal of Formal Logic, vol. 20, no. 2, p. 438-450, 1979.

[8] Hughes C. E. Two Variable Implicational Calculi of Prescribed Many-One Degrees of Unsolvability. // Journal of Symbolic Logic, vol. 41, no. 1, p. 39-44, 1976.

[9] Hughes C. E., Singletary W. E. Triadic partial implicational propositional calculi. // Zeitschriftfdr für mathematische Logik und Grundlagen der Mathematik, vol. 21, p. 2128, 1975 .

[10] Linial S., Post E. L. Recursive unsolvability of the deducibility, Tarski's comleteness, and independence of axioms problems of the propositional calculus. // Bulletin of the American Mathematical Society, vol. 55, p. 50, 1949.

[11] Minsky M. L. Computation: Finite and Infinite Machines. - Upper Saddle River, NJ, USA, Prentice-Hall, Inc., 1967.

[12] Popov S. Nondecidable intermediate calculus. // Algebra and Logic, vol. 20, no. 6, p. 424-461, 1981. 
[13] Rybakov M. N. Complexity of intuitionistic and Visser's basic and formal logics in finitely many variables. // Advances in Modal Logic, p. 393-411, 2006.

[14] Shehtman V. B. On incomplete propositional logics. // Soviet Mathematics Doklady, vol. 18, p. 985-989, 1977.

[15] Shehtman V. B. An undecidable superintuitionistic propositional calculus. // Soviet Mathematics Doklady, vol. 240, no. 3, p. 549-552, 1978. (Russian).

[16] Shehtman V. B. Undecidable propositional calculi. // Problems of Cybernetics, vol. 75, p. 74-116, 1982. (Russian).

[17] Skvortsov D. P. One superintuitionistic calculus of propositions. // Algebra and Logic, vol. 24, no. 2, p. 119-125, 1985.

[18] Sobolev S. K. On the finite approximability of superintuitionistic logics. // Mathematics of the USSR, vol. 31, p. 257-268, 1977. (Russian). 